\title{
GRB spectral parameter modeling
}

\author{
Gregory D. Fleishman ${ }^{1,2}$ and Fedor A. Urtiev ${ }^{3}$ \\ ${ }^{1}$ New Jersey Institute of Technology, Newark, NJ 07102, USA \\ ${ }^{2}$ Ioffe Institute, St. Petersburg 194021, Russia \\ email: gfleishm@njit.edu \\ ${ }^{3}$ State Polytechnical University, St.Petersburg, 195251, Russia \\ email: zigzagworld@rambler.ru
}

\begin{abstract}
Fireball model of the gamma-ray bursts (GRBs) predicts generation of numerous internal shocks, which efficiently accelerate charged particles and generate relatively small-scale stochastic magnetic and electric fields. The accelerated particles diffuse in space due to interaction with the random waves and so emit so called Diffusive Synchrotron Radiation (DSR) in contrast to standard synchrotron radiation they would produce in a large-scale regular magnetic fields. In this contribution we present key results of detailed modeling of the GRB spectral parameters, which demonstrate that the non-perturbative DSR emission mechanism in a strong random magnetic field is consistent with observed distributions of the Band parameters and also with cross-correlations between them.
\end{abstract}

Keywords. acceleration of particles, shock waves, turbulence, galaxies: jets, radiation mechanisms: non-thermal, magnetic fields

\section{Introduction}

The fireball model of the gamma-ray burst (GRB) suggests that a central engine produces a number of interacting relativistic shock waves, whose interactions, in the collisionless case, result in generation of fluctuating electromagnetic fields and acceleration of charged particles up to high energies. It is well established by now that the magnetic and electric fields produced in the shock interactions have often a significant random component at various spatial scales. As a result, the shock-accelerated charged particles moving through a plasma with random electromagnetic fields experience random Lorenz forces and so follow random paths representing a kind of spatial diffusion. Accordingly, the particles produce a "diffusive radiation" whose spectra depend on the type of the field (magnetic or electric) and on spectral energy distribution of the field over the spatial scales (Toptygin \& Fleishman 1987, Fleishman 2006, Fleishman \& Toptygin 2007a, Fleishman \& Toptygin 2007b, Reville \& Kirk 2010).

Individual spectra of the prompt GRB emission are typically well fitted by a phenomenological Band function (Band et al. 1993), which consists of low-energy (spectral index $\alpha$ ) and high-energy (spectral index $\beta$ ) power-law regions smoothly linked at a break energy $E_{\mathrm{br}}$. The DSR was shown (Fleishman 2006) to produce spectra consistent with those observed typically from the GRBs (Band et al. 1993). It had yet been unclear, however, if the DSR spectra are naturally consistent with observed distributions of the GRB spectral parameters (Preece et al. 2000; Kaneko et al. 2006) and what ranges of physical GRB parameters are needed to reconcile the theoretical spectra with the observed ones. In this conference contribution we present results of the modeling of GRB prompt emission generation by DSR in relativistically expanding GRB jets presented in greater detail by Fleishman \& Urtiev (2010). 

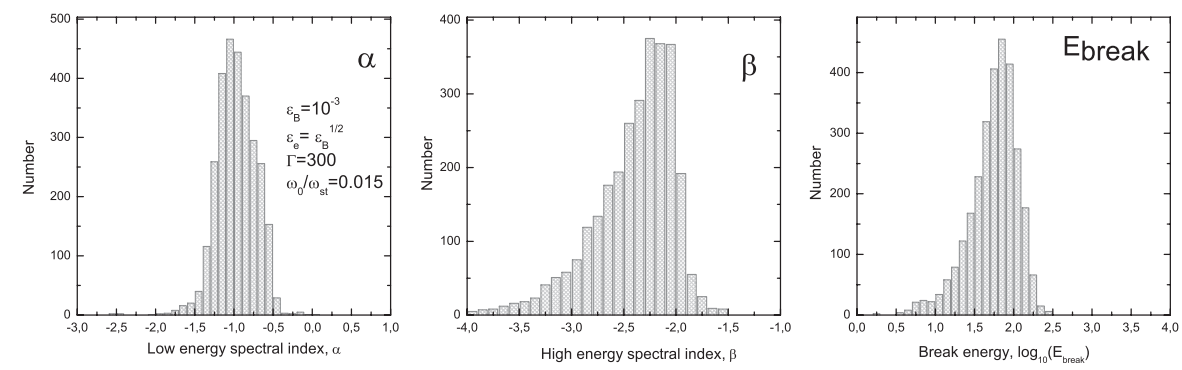

Figure 1. Example of the model Band parameter $\left(\alpha, \beta\right.$, and $\left.E_{\mathrm{br}}\right)$ distributions obtained within the DSR model with strong random magnetic field.

\section{Model}

Formulation. Adopting a general internal shocks/fireball concept we accept that a single binary collision of relativistic internal shocks results in a single episode of the GRB prompt emission (Fleishman \& Urtiev 2010). Microscopically, this shock-shock interaction produces high levels of random magnetic and/or electric fields and accelerates the charged particles up to large ultrarelativistic energies; these particles interact with the random fields to generate the gamma-rays. Although there are some common general properties of all cases of relativistic shock interactions, each shock-shock collision is, nevertheless, unique in terms of combination of the physical parameters involved. Accordingly, we adopt a set of standard (mean) parameters appropriate to account for the most global GRB properties, and then consider if a reasonable scatter of those standard parameters is capable of reproducing more detailed properties of the considered class of events as a whole - the statistical distributions of the GRB spectral parameters and cross-correlations between them. To do so, we considered a number of different emission models including the standard synchrotron radiation and DSR regimes in case of either weak or strong random magnetic field. The spectral slopes and breaks depend on both the emission mechanism and combination of physical parameters affecting the radiation spectra within a given mechanism. Thus, the goal of the modeling is to establish if there exists a parametric space making one or another theoretical model compatible with the observational data on the GRB spectral properties.

Results. Fleishman \& Urtiev (2010) conclude that the DSR model with the weak random magnetic field (jitter regime), either perturbative or non-perturbative, cannot offer a consistent fit to the observed $\alpha$ histogram. This complies with independent criticisms of the jitter regime: Kumar \& McMahon (2008) noticed that it may imply an unrealistically high level of inverse Compton emission, while Kirk \& Reville (2010) argued that the jitter case seems to be in contradiction with the required high efficiency of the particle acceleration at the shocks, so strong magnetic fluctuations are needed to self-consistently accelerate electrons up to the gamma-ray producing energies.

Thus, having the weak random field model (jitter regime) rejected, we turn now to analysis of the strong random field case. According to Fleishman (2005), Fleishman \& Bietenholz (2007), and Reville \& Kirk (2010), new asymptotes arise in this case, which can yield broader $\alpha$ distribution. In this strong-field regime, the model $\alpha$ distribution depend on adopted $\nu$ distribution, which, within the adopted model, is straightforwardly linked to the $\beta$ distribution, because $\beta=-\nu-1$. The corresponding model results (Fig. 1) are in a remarkable agreement with the observations. Indeed, the $\alpha$ histogram is a symmetric one, it displays a peak at the right place, $\alpha=-1$, and its bandwidth is comparable to that of the observed histogram. The $\beta$ histogram almost repeats the observed one, displaying the correct asymmetric shape and the peak at the right place, 

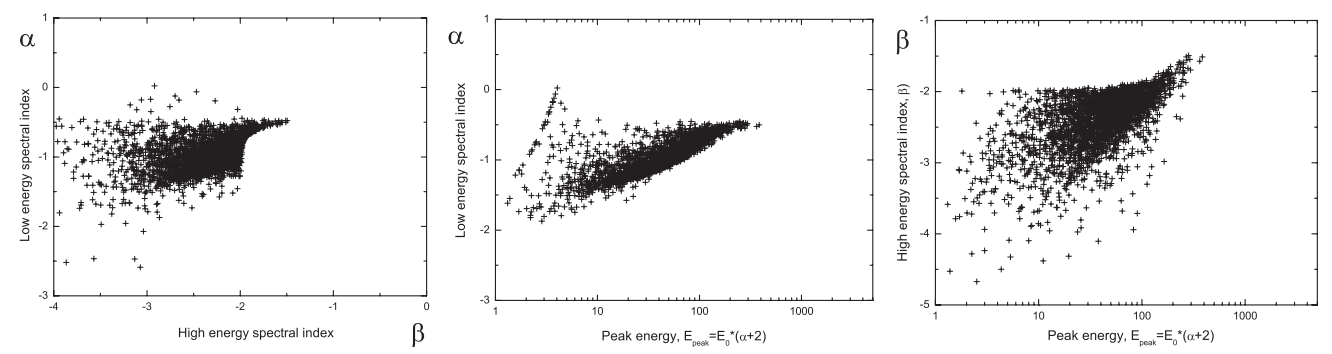

Figure 2. Cross-correlation of the model Band spectral parameters $\alpha$ and $\beta$ (left); $\alpha$ and $E_{\mathrm{peak}}=(\alpha+2) E_{\mathrm{br}}($ middle $) ;$ and $\beta$ and $E_{\mathrm{peak}}=(\alpha+2) E_{\mathrm{br}}$ (right).

$\beta=-2.2$. The $E_{\mathrm{br}}$ histogram also agrees with the observed one rather well displaying correct shape and bandwidth.

The cross-correlations between the spectral parameters derived from the model (Fig. 2) are to be compared with fig. 31 from Kaneko et al. (2006). Like in the observation, the spectral indices $\alpha$ and $\beta$ are not highly correlated, although in the model plot the region of $-0.5<\alpha<0$ is underpopulated compared with the observed plot (Kaneko et al. 2006). Two other plots are in remarkable agreement with the observed cross-correlation plots, presented in Kaneko et al. (2006).

\section{Conclusions}

We conclude that the developed model is naturally capable of reproducing both the Band parameter histograms and their cross-correlations, which is a remarkable success of the non-perturbative DSR model in the presence of strong random magnetic field.

\section{Acknowledgments}

This work was supported in part by the Russian Foundation for Basic Research, grants No. 08-02-92228, 09-02-00226, 09-02-00624. We have made use of NASA's Astrophysics Data System Abstract Service.

\section{References}

Band, D. et al. 1993, ApJ, 413, 281

Fleishman, G. D. 2005, ArXiv e-prints: astro-ph/0510317

-. 2006, ApJ, 638, 348

Fleishman, G. D. \& Bietenholz, M. F. 2007, MNRAS, 376, 625

Fleishman, G. D. \& Toptygin, I. N. 2007a, MNRAS, 381, 1473

-. 2007b, Phys. Rev. E, 76, 017401

Fleishman, G. D. \& Urtiev, F. A. 2010, MNRAS, 406, 644

Kaneko, Y. et al. 2006, ApJS, 166, 298

Kirk, J. G. \& Reville, B. 2010, ApJ, 710, L16

Kumar, P. \& McMahon, E. 2008, MNRAS, 384, 33

Preece, R. D. et al. 2000, ApJS, 126, 19

Reville, B. \& Kirk, J. G. 2010, ArXiv e-prints: astro-ph.HE/1010.0872; this conf. proc.

Toptygin, I. N. \& Fleishman, G. D. 1987, Astrophys. Space. Sci., 132, 213 